\title{
BMJ Open Decision analytic model of the diagnostic pathways for patients with suspected non-alcoholic fatty liver disease using non-invasive transient elastography and multiparametric magnetic resonance imaging
}

\author{
Laurence Blake, Rui V Duarte, Carole Cummins
}

To cite: Blake L, Duarte RV, Cummins C. Decision analytic model of the diagnostic pathways for patients with suspected non-alcoholic fatty liver disease using non-invasive transient elastography and multiparametric magnetic resonance imaging. $B M J$ Open 2016;6:e010507. doi:10.1136/bmjopen-2015010507

- Prepublication history for this paper is available online. To view these files please visit the journal online (http://dx.doi.org/10.1136/ bmjopen-2015-010507)

LB and RVD contributed equally.

Received 9 November 2015 Revised 30 August 2016 Accepted 31 August 2016

Institute for Applied Health Research, University of Birmingham, Birmingham, UK

Correspondence to Dr Rui V Duarte; r.duarte@bham.ac.uk

\section{ABSTRACT}

Objectives: The mortality associated with liver disease continues to increase, despite the improvements implemented in the UK healthcare as does the prevalence of non-alcoholic fatty liver disease (NAFLD), given the escalating prevalence of obesity. The currently available methods to assess and monitor the stage of liver disease present several limitations. Recently, multiparametric MRI has been developed to address these limitations. The aim of this study is to develop a decision analytic model for patients with suspected NAFLD, to investigate the effect of adding multiparametric MRI to the diagnostic pathway.

Perspective: The model takes the perspective of the UK National Health Service (NHS) as the service provider.

Methods: A simple decision-tree model was developed to compare the costs associated with 3 diagnostic pathways for NAFLD that use non-invasive techniques. First, using transient elastography alone; second, using multiparametric MRI as an adjunct to transient elastography and third, multiparametric MRI alone. The model was built to capture these clinical pathways, and used to compare the expected diagnostic outcomes and costs associated with each.

Results: The use of multiparametric MRI as an adjunct to transient elastography, while increasing screening costs, is predicted to reduce the number of liver biopsies required by about $66 \%$. Used as the sole diagnostic scan, there remains an expected $16 \%$ reduction in the number of biopsies required. There is a small drop in the overall diagnostic accuracy, as in the current model, liver biopsy is presumed to give a definitive diagnosis.

Conclusions: The inclusion of multiparametric MRI, either as an adjunct to or replacement of transient elastography, in the diagnostic pathway of NAFLD may lead to cost savings for the NHS if the model presumptions hold. Further high-quality clinical evidence and cost data are required to test the model's predictions.

\section{Strengths and limitations of this study}

- This is the first study to evaluate the costs associated with the inclusion of a new method to assess liver disease in the diagnostic pathway of patients with suspected non-alcoholic fatty liver disease.

- Potential cost savings to the NHS have been identified by the use of multiparametric MRI as an adjunct to or replacement of transient elastography if the model presumptions hold.

- The current decision analytic model compares only the diagnostic pathways; it does not consider the consequences of any diagnosis and does not follow the progression of liver disease in individuals.

- Additional high-quality clinical evidence and cost data are necessary to develop and test the model's predictions further.

\section{INTRODUCTION}

Liver disease refers to any disorder of the liver that leads to a reduction in its functioning. There are several types of liver disease, including sequelae of hepatitis, alcoholic liver disease (ALD) and non-alcoholic fatty liver disease (NAFLD). ALD and NAFLD have similar pathological spectra and disease may progress through simple hepatic steatosis to steatohepatitis, cirrhosis and hepatocellular carcinoma. ${ }^{12}$ The clinical differentiation between ALD and NAFLD is usually performed by taking a history of a patient's alcohol intake combined with laboratory and imaging examinations. Patients with non-alcoholic steatohepatitis (NASH) usually exhibit more advanced fatty degeneration of liver cells than those with alcoholic steatohepatitis and the inflammatory 
infiltrate in NASH is somewhat less pronounced than in alcoholic steatohepatitis. ${ }^{2}$

Improvements made in UK healthcare have resulted in a decrease in mortality rates for most health conditions, including heart disease, endocrine or metabolic disease, respiratory disease and diabetes. ${ }^{3}$ Liver disease is the exception. The standardised mortality rate has increased by almost $500 \%$ since 1970 in patients younger than 65 years. ${ }^{3}$ Liver disease accounts for 62000 years of working life lost every year; only ischaemic heart disease (74 000 years) and self-harm (71 000 years) lead to a greater premature loss of life. ${ }^{4}$

Between 1988 and 2008, the prevalence of chronic liver disease caused by hepatitis B virus, hepatitis $\mathrm{C}$ virus and ALD has remained stable. ${ }^{5}$ During the same period, the prevalence of NAFLD increased from $5.51 \%$ to $11.01 \% .^{5}$ It is expected that the prevalence of NAFLD will continue to increase, given the escalating prevalence of obesity; with projections to the year 2030 estimating a $33 \%$ increase in obesity and a $130 \%$ increase in severe obesity. ${ }^{6}$

Fatty liver (ie, simple steatosis) was believed to be a benign condition with no or minimal rate of progression. However, recent evidence suggests that a substantial proportion of patients (28-32\%) with simple steatosis progress towards NASH and fibrosis within a 3 to 4-year period. ${ }^{7-9}$ In most patients, NASH develops on a background of diabetes or impaired glucose tolerance in the long term. ${ }^{10}$ Progression to cirrhosis, hepatocellular carcinoma and increased mortality has been reported for patients with NASH. ${ }^{11} 12$

It is important, therefore, to detect fatty liver disease at its early stages before progression into NASH, a cirrhotic stage or liver cancer. The early stages of NAFLD can be managed and may regress if lifestyle advice is provided and followed. Weight reduction has been found to be associated with non-progressive disease. ${ }^{9}$ The early detection of NAFLD is important to establish an effective course of treatment, and has the potential to reduce the economic burden of liver disease and save lives. ${ }^{13}$ Recent EASL-EASD-EASO clinical practice guidelines have recommended that all individuals with persistently abnormal liver enzymes or steatosis should be screened for NAFLD. ${ }^{14}$

\section{Liver biopsy}

Liver biopsy is currently considered as the reference standard for the diagnosis of liver disease. Liver biopsy is nevertheless imperfect when used to assess the extent of disease progression in terms of fibrotic transformation of liver tissue. This is because it allows examination of only a very small area of the liver, potentially missing the disease as changes within the liver can be patchy. In addition, there is variability in histological interpretation depending on the individual pathologist's experience. ${ }^{15} 16$ Liver biopsy is invasive and associated with a risk of haemorrhagic complications. It can also be painful and stressful for the patient as well as time- consuming. It is a relatively costly procedure and has a low level of diagnostic performance for early stages of fibrosis. ${ }^{17}{ }^{18}$ Liver biopsy may cause anxiety in patients, and has been found to be painful in up to $30 \%$ of cases. ${ }^{19}$ A recent willingness to pay evaluation found that most patients $(75 \%)$ who had undergone a liver biopsy (publicly funded in British Columbia) would be willing to self-pay for transient elastography (not publicly funded in British Columbia). ${ }^{20}$ The majority of patients preferred the non-invasive transient elastography method, as it was associated with less discomfort during and after the scan, and no feelings of anxiety after the procedure was explained. ${ }^{20}$ Only those patients with unknown liver disease were found to prefer liver biopsy. There is a need in the diagnostic and monitoring pathway for non-invasive methods to assess and monitor the stage of liver disease.

\section{Transient elastography}

Transient elastography is a non-invasive method to assess hepatic fibrosis using ultrasound to measure the velocity of an elastic shear wave transmitted through the liver and assess liver stiffness. ${ }^{21}$ It is a painless test for which sedation is not required, it is significantly less expensive than liver biopsy and it has not been associated with any adverse treatment-effects. ${ }^{16}$ However, despite being widely used, the cut-off values of liver stiffness for the different stages of liver fibrosis are not well established. ${ }^{22}$ Using transient elastography, significant variations in liver stiffness measurements related to operator and patient factors rather than to disease progression have been observed. ${ }^{23}$ The variations in cut-off values and measurements limit the effectiveness of transient elastography for monitoring and assessing the progression of liver fibrosis. ${ }^{23}$ In addition, transient elastography has a high failure rate, particularly among obese patients. The reported failure rates vary widely, ranging from $4.5 \%$ in a cohort of patients with chronic liver disease ${ }^{24}$ to $41 \%$ in a cohort of patients with BMI of $35 \mathrm{~kg} / \mathrm{m}^{2}$ or higher. ${ }^{9}$ A 5-year prospective study of 13369 examinations of patients with suspected chronic liver disease reported an average failure rate for transient elastography of $18.4 \%{ }^{25}$ The main factors influencing reliability were limited operator experience and obesity, particularly increased waist circumference. Subgroup analysis in this study found the failure rate ranging from $12 \%$ $(\mathrm{BMI}<25)$ to $53 \%(\mathrm{BMI}>40) .{ }^{25}$ Failure rates for transient elastography are higher for obese patients as the ultrasound wave used by the probe can be strongly attenuated by fatty tissue. ${ }^{26}$ This limitation is important as obese patients have an increased risk of liver disease progression.

\section{Multiparametric magnetic resonance imaging}

Multiparametric magnetic resonance imaging (MRI) is a new non-invasive technique designed to diagnose liver fibrosis. It consists of software (LiverMultiScan) that enables the assessment of multiparametric liver data (ie, 
fat, iron and fibrosis) based on an MRI scan. The first study on this technology reported an average scan time of $23 \mathrm{~min}$ and demonstrated that multiparametric MRI can quantify hepatic fibrosis, iron and steatosis. ${ }^{27}$ Transverse abdominal $\mathrm{T} 1$ and $\mathrm{T} 2 *$ MR maps, corresponding to segment 8 of the liver, are acquired. ${ }^{27}$ The majority of percutaneous liver biopsies are taken from this area. Once the image is acquired, an operator defines a region of interest of the liver lobe, away from vascular and biliary structures. The image is analysed remotely, removing the need for interpretation by a radiologist, potentially reducing the time needed for scan results and costs. The software generates a report for the clinician, with analyses of fat, iron and fibrosis levels in the liver. Multiparametric MRI has been included as the only liver imaging test in the UK Biobank study.

\section{Aim of the study}

The aim of this study was to develop a preliminary decision analytic model of the diagnostic pathways for patients with suspected NAFLD using two non-invasive methods: (1) transient elastography and (2) multiparametric MRI. Such a model could indicate the potential value of investment in further research and inform the design of such research.

\section{METHODS}

\section{Modelling methodology}

A simple decision-tree model was constructed in Excel to compare the costs associated with three diagnostic pathways for NAFLD that use non-invasive techniques. First, using transient elastography alone, then using multiparametric MRI as an adjunct to transient elastography and finally, using multiparametric MRI alone (figure 1). The chosen pathways were based on current clinical practice according to clinical advice. The model was built to capture these clinical pathways, and used to compare the expected diagnostic outcomes and costs associated with each.

For the base case model, it is presumed that the diagnostic pathways as set out in figure 1 are followed exactly by all patients. In practice, these pathways, and the decision whether to take a liver biopsy at any stage, may vary between individual patients depending on other indications or clinical opinion.

The first patient pathway uses transient elastography as the first-line non-invasive diagnosis. Patients whose test results are within the normal range are referred back to their general practitioner and no further immediate tests are carried out. Patients giving a positive test move on to the next stage in the diagnostic pathway, which in this case is a confirmatory liver biopsy. Patients for whom the test failed also move on to the next stage of liver biopsy.

In the second pathway, multiparametric MRI is introduced as a second line, non-invasive diagnostic tool for those patients for whom the transient elastography either gave a positive diagnosis or failed, and who would otherwise have had a liver biopsy at this stage. For those with a positive transient elastography, a further positive diagnosis with multiparametric MRI is considered as confirmatory with no further tests necessary, whereas a contradictory negative test or a failure results in a liver biopsy. For those patients for whom the initial transient elastography failed, multiparametric MRI becomes the first-line diagnosis whereby test outcomes are treated as with transient elastography alone. That is, a normal result requires no immediate further action, a positive result would require a confirmatory biopsy and a second failure would be followed by a diagnostic biopsy.

In the final pathway, multiparametric MRI replaces transient elastography as the first-line diagnostic tool with test outcomes treated in the same way.

\section{Model parameters}

A hypothetical cohort of 1000 patients presenting with suspected NAFLD was modelled. Initial clinical suspicion would be based on laboratory findings and the absence of other causes of liver disease. The estimated prevalence for the successive stages of fibrosis in the cohort was taken from a recent Health Technology Assessment (HTA) ${ }^{28}$ In their analysis, 48 studies were used to assess the sensitivity and specificity of a number of diagnostic tools at successive thresholds of liver fibrosis. Overall prevalence at each threshold was calculated from the populations in the included studies. The median prevalence (minimum-maximum) of fibrosis stages F1-F4 in the studies identified, as well as additional model parameters, are presented in table 1 . The median prevalence at each threshold was taken to calculate prevalence for each level of fibrosis in the modelled population.

In the model, the sensitivity and specificity of transient elastography at each threshold, as calculated in the HTA, were used to predict the proportion of positive and negative test results. For multiparametric MRI, sensitivity and specificity for any level of fibrosis were taken from Banerjee et al. ${ }^{27}$ For those in the modelled cohort with liver fibrosis, the relevant sensitivities of the tests were used to predict the rates for true positives and false negatives, while for those without fibrosis, the specificities were used to predict the rates of false positives and true negatives. Rates for test failures for multiparametric MRI were provided by the manufacturer.

\section{Costs}

The model takes the perspective of the UK National Health Service (NHS) as the service provider. The costs for transient elastography and liver biopsy were derived from the HTA report ${ }^{28}$ and inflated from 2012 to 2014 prices using the Personal Social Services Research Unit inflator. ${ }^{29}$ A price for the multiparametric MRI procedure is not currently available. For the base case analysis, we presumed the cost of multiparametric MRI to be the same as transient elastography. Cost-effectiveness thresholds for multiparametric MRI were evaluated for 


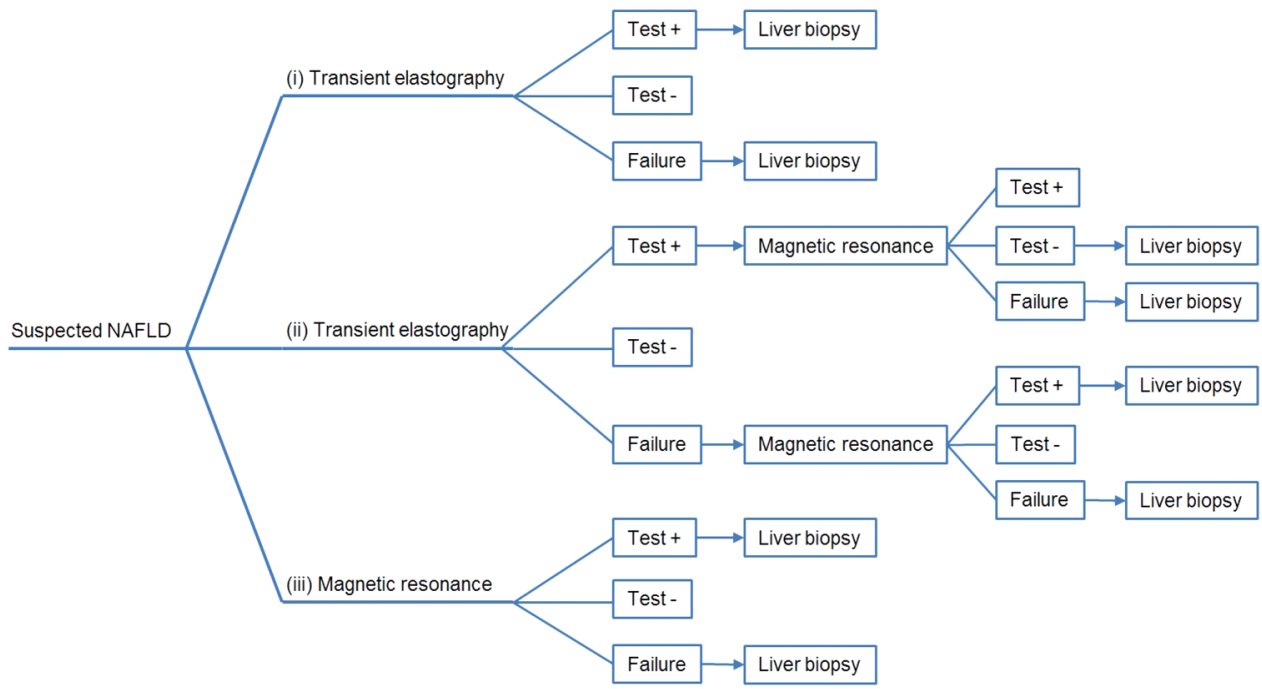

Figure 1 Decision analytic model of diagnostic pathways.

\begin{tabular}{|c|c|c|}
\hline Model parameter & & Source \\
\hline \multicolumn{3}{|l|}{ NAFLD prevalence } \\
\hline Fibrosis stage & Median (minimum-maximum) & \multirow{5}{*}{ Crossan et $a R^{8}$} \\
\hline $\mathrm{F} 1$ & $0.588(0.367-0.814)$ & \\
\hline F2 & $0.319(0.119-0.526)$ & \\
\hline F3 & $0.186(0.050-0.440)$ & \\
\hline F4 & $0.128(0.039-0.907)$ & \\
\hline \multicolumn{3}{|c|}{ Sensitivity of transient elastography for diagnosis of NAFLD } \\
\hline Fibrosis stage & Summary sensitivity $(95 \% \mathrm{Cl})$ & \multirow{5}{*}{ Crossan et $a R^{8}$} \\
\hline$F \geq 1$ & $0.87(0.81$ to 0.92$)$ & \\
\hline$F \geq 2$ & $0.79(0.72$ to 0.85$)$ & \\
\hline$F \geq 3$ & $0.82(0.74$ to 0.88$)$ & \\
\hline$F \geq 4$ & 0.96 (0.83 to 0.99$)$ & \\
\hline \multicolumn{3}{|c|}{ Specificity of transient elastography for diagnosis of NAFLD } \\
\hline Fibrosis stage & Summary specificity $(95 \% \mathrm{Cl})$ & \multirow{5}{*}{ Crossan et $a f^{28}$} \\
\hline $\mathrm{F} \geq 1$ & $0.76(0.57$ to 0.88$)$ & \\
\hline $\mathrm{F} \geq 2$ & $0.76(0.71$ to 0.80$)$ & \\
\hline $\mathrm{F} \geq 3$ & $0.84(0.78$ to 0.89$)$ & \\
\hline $\mathrm{F} \geq 4$ & 0.89 (0.85 to 0.92$)$ & \\
\hline \multicolumn{3}{|c|}{ Sensitivity of multiparametric MRI for diagnosis of NAFLD } \\
\hline Any fibrosis & 0.86 & Banerjee et $a f^{7}$ \\
\hline \multicolumn{3}{|c|}{ Specificity of multiparametric MRI for diagnosis of NAFLD } \\
\hline Any fibrosis & 0.93 & \multirow[t]{2}{*}{ Banerjee et $a^{7}$} \\
\hline Failure rates & Base case (range) & \\
\hline Transient elastography & $18.4 \%(12-50 \%)$ & Castéra et $a^{5}$ \\
\hline Multiparametric MRI & $5 \%(2.5-10 \%)$ & Manufacturer data \\
\hline \multicolumn{3}{|l|}{ Costs } \\
\hline Transient elastography & $£ 52.44$ & \multirow{2}{*}{ Crossan et $a P^{8}$} \\
\hline Liver biopsy & $£ 983.70$ & \\
\hline
\end{tabular}

each of the diagnostic pathways with this diagnosis option. Probabilistic sensitivity analysis was conducted using the sensitivity and specificity of transient elastography. Given the short modelling horizon of the diagnostic pathways, no costs were discounted.

\section{RESULTS}

Diagnostic pathway

Using transient elastography alone with the median values for sensitivity and specificity, the model suggests that for the cohort of 1000 patients with suspected 
NAFLD, there would be 496 positive and 319 negative test results. With 184 failures, 680 patients would move to the next diagnostic level; which in this case is a liver biopsy. Based on the prevalence of fibrosis, and the specificity of transient elastography, 64 patients with fibrosis would continue undiagnosed, giving a diagnostic accuracy for this pathway of $93.6 \%$ if liver biopsy is presumed to give a definitive diagnosis. Introducing multiparametric MRI as a second-line diagnostic tool before liver biopsy requires a further 680 multiparametric MRI tests for those patients thus indicated, but is predicted to more than halve the total number of liver biopsies required to 254. With the reduced number of biopsies, the overall diagnostic accuracy falls to $91.6 \%$, with 78 patients with fibrosis remaining undiagnosed and 5 patients without fibrosis receiving an incorrect positive diagnosis. Using multiparametric MRI instead of transient elastography would be expected to yield 508 positive and 442 negative test results. With 50 failures, 558 liver biopsies would then be indicated. The diagnostic accuracy for this pathway is $92.2 \%$, with 78 undiagnosed cases of fibrosis.

\section{Cost analysis}

For multiparametric MRI to be a cost-efficient addition in the diagnosis of NAFLD, any increase in costs associated with its use, either as an adjunct to or instead of transient elastography, needs to be compensated for by a reduction in the number of biopsies needed. As a reference point, if multiparametric MRI were to cost the same as transient elastography, that is, $£ 52.44$, the results outlined above would give the cost outcomes as summarised in table 2.

When using multiparametric MRI as an adjunct to transient elastography, the cost of the expected extra 680 tests is more than offset by the savings made by the reduction in the number of biopsies required. When used instead of transient elastography, the cost of testing remains the same, and there is some reduction in the number of expected biopsies, due to a lower failure rate and better diagnostic accuracy (mainly a better selectivity resulting in a lower rate of false positives).

\section{Threshold and sensitivity analysis}

The expected cost savings to be made in the two scenarios that use multiparametric MRI suggest that there is an opportunity to increase the price. When used as a second-line diagnosis after transient elastography, the use of multiparametric MRI remains cost-effective up to $£ 616$ per test. This figure reflects the potential cost savings that could be made by performing these two types of scans before considering a biopsy. When used as the sole non-invasive diagnostic tool prior to liver biopsy, multiparametric MRI remains a cost-effective replacement for transient elastography up to a cost of $£ 173$ per test. This figure is lower than the previous threshold as in this scenario, there is again just one scanning method used before a possible biopsy.

\section{Probabilistic sensitivity analysis}

The HTA report used the included studies to calculate the sensitivity and specificity of transient elastography, reporting mean values with $95 \%$ CIs. ${ }^{28}$ Probabilistic sampling was performed on these distributions to assess the robustness of the deterministic estimate of costeffectiveness when using transient elastography and multiparametric MRI in combination. The results of the random sampling show an SD in the cost difference of $£ 42$ per test, suggesting that there is a $95 \%$ probability of this strategy remaining cost-effective up to a price threshold of $£ 547$.

\section{Threshold analysis}

Setting the sensitivity and specificity of transient elastography at the lower and upper 95\% CIs gives break-even prices for multiparametric MRI when used in conjunction with transient elastography of $£ 558$ and $£ 659$, respectively. The price of multiparametric MRI therefore needs to be reduced if the performance of transient elastography is set to the most pessimistic levels. This is because transient elastography gives an increase in the proportion of positive results (from $41 \%$ to 53\%) at this lower diagnostic accuracy. These patients then go on to multiparametric MRI and, as their transient elastography results are less accurate, they are more likely to be contradicted by multiparametric MRI. It is these patients with contradictory results who then go on for a liver biopsy. The model predicts that the percentage of the original cohort in this category would rise from $9.5 \%$ at the upper confidence level to $18 \%$ at the lower confidence level. Thus, a more accurate transient

Table 2 Base case results

\begin{tabular}{|c|c|c|c|c|c|c|}
\hline & \multicolumn{2}{|c|}{ Transient elastography } & \multicolumn{2}{|c|}{$\begin{array}{l}\text { Transient elastography } \\
\text { plus multiparametric } \\
\text { MRI }\end{array}$} & \multicolumn{2}{|c|}{ Multiparametric MRI } \\
\hline & Number & Cost & Number & Cost & Number & Cost \\
\hline Transient elastography tests & 1000 & $£ 52440$ & 1000 & $£ 52440$ & 0 & $£ 0$ \\
\hline Multiparametric MRI tests & 0 & $£ 0$ & 680 & $£ 35684$ & 1000 & $£ 52440$ \\
\hline Liver biopsies & 679 & $£ 669374$ & 254 & $£ 249902$ & 558 & $£ 548702$ \\
\hline Total cost & \multicolumn{2}{|c|}{$£ 721814$} & \multicolumn{2}{|c|}{$£ 338026$} & \multicolumn{2}{|c|}{$£ 601142$} \\
\hline
\end{tabular}


elastography means fewer contradictory results, with fewer resultant biopsies. In the third treatment pathway of multiparametric MRI alone, the corresponding breakeven costs are $£ 203$ for the lower CI, and $£ 156$ for the upper CI.

\section{Transient elastography and multiparametric MRI failure rate}

Transient elastography and multiparametric MRI can fail or give unreliable results. This is caused by patient characteristics, technical issues with the equipment or operator inexperience. As NAFLD is associated with higher BMI, it might be expected that the failure rate for transient elastography in NAFLD patients would be higher than the $18.4 \%$ average reported in the Castéra et $a t^{25}$ study. However, with a lack of evidence to quantify any difference in average BMI of the two patient groups, and the subsequent effect on transient elastography failure rates, the figure of $18.4 \%$ was used in the model as a conservative estimate of the baseline failure rate. For multiparametric MRI, BMI is less of an issue, with failures related more to technical issues. Trials by the manufacturer have indicated a failure rate in the range of $\sim 2.5-5 \%$ associated with the use of multiparametric MRI.

With these figures in mind, tables 3 and 4 show an illustrative range of failure rates for transient elastography and multiparametric MRI, with the estimated breakeven cost of multiparametric MRI when used as an adjunct to or replacement for transient elastography, respectively.

In the first scenario, as the failure rate of transient elastography increases, a higher proportion of patients move on to the second line diagnosis, with an associated increase in the total number of biopsies. With the extra cost of these biopsies, the break-even price of multiparametric MRI decreases. In the second scenario, as the failure rate of transient elastography goes up, the breakeven price of multiparametric MRI also goes up, as it is now replacing a decreasingly reliable transient elastography. In both scenarios, the break-even price of multiparametric MRI decreases with increased failures, as any extra failures at this stage mean extra liver biopsies.

\section{Multiparametric MRI as the sole diagnostic test}

For the third diagnostic pathway in figure 1, multiparametric MRI replaces transient elastography as the firstline diagnostic test. In the modelled base-case for this

Table 3 Break-even cost of multiparametric MRI when used as an adjunct to transient elastography

\begin{tabular}{|c|c|c|c|c|}
\hline \multirow{2}{*}{$\begin{array}{l}\text { Multiparametric } \\
\text { MRI failure rate }\end{array}$} & \multicolumn{4}{|c|}{$\begin{array}{l}\text { Transient elastography } \\
\text { failure rate }\end{array}$} \\
\hline & $12 \%$ & $18 \%$ & $35 \%$ & $50 \%$ \\
\hline $2.5 \%$ & $£ 654$ & $£ 638$ & $£ 581$ & $£ 543$ \\
\hline $5 \%$ & $£ 638$ & $£ 617$ & $£ 567$ & $£ 529$ \\
\hline $10 \%$ & $£ 604$ & $£ 585$ & $£ 537$ & $£ 501$ \\
\hline
\end{tabular}

Table 4 Break-even cost of multiparametric MRI when used as a replacement for transient elastography

\begin{tabular}{lllll}
\hline Multiparametric & \multicolumn{4}{l}{ Transient elastography failure rate } \\
\cline { 2 - 5 } MRI failure rate & $\mathbf{1 2 \%}$ & $\mathbf{1 8 \%}$ & $\mathbf{3 5 \%}$ & $\mathbf{5 0 \%}$ \\
\hline $2.5 \%$ & $£ 159$ & $£ 221$ & $£ 248$ & $£ 306$ \\
$5 \%$ & $£ 148$ & $£ 210$ & $£ 237$ & $£ 294$ \\
$10 \%$ & $£ 145$ & $£ 187$ & $£ 214$ & $£ 271$ \\
\hline
\end{tabular}

scenario, as with transient elastography, patients receiving a positive diagnosis go on for a confirmatory biopsy to assess the nature and extent of any fibrosis. If it can be shown that multiparametric MRI is able to match the diagnostic accuracy of liver biopsy in this role, then there is the potential for it to replace biopsy as the definitive diagnosis of liver fibrosis. Incorporating this possibility into the model reduces the number of biopsies by 508 per 1000 patients, the expected number of positive multiparametric MRI tests; leaving biopsies for just the $5 \%$ of patients for whom the multiparametric MRI fails. Obviating the need for biopsies for those patients with positive multiparametric MRI reduces the total testing costs to $14 \%$ of those in the first scenario of transient elastography backed up with liver biopsy. This means that the price of multiparametric MRI could remain cost-effective up to a price of $£ 672$, if used as the sole diagnostic test replacing the combination of transient elastography and liver biopsy. Removing biopsy as the second-line test inevitably has an effect on the overall diagnostic accuracy of this pathway, reducing the rate of correct diagnoses to $89 \%$, with 78 cases of fibrosis remaining undiagnosed and 27 patients without fibrosis receiving a false-positive test result.

\section{DISCUSSION}

This study proposes that the current NAFLD diagnostic pathway may become more cost-efficient with the inclusion of multiparametric MRI either as an adjunct to or replacement of transient elastography. The use of multiparametric MRI as an adjunct to transient elastography has the potential to reduce the number of liver biopsies by $66 \%$ while as a replacement would result in a decrease in the number of biopsies needed of $16 \%$. A small drop in predicted diagnostic accuracy is predicted, but this is inevitable because some biopsies are avoided, and these are presumed, for our model, to be $100 \%$ accurate. Acquisition of further clinical evidence is required to confirm whether the use of multiparametric MRI as an adjunct to or replacement of transient elastography can result in cost savings for the NHS. The current study presents a preliminary decision analytic model, which can be adapted and developed as more evidence becomes available.

Given the increasing prevalence of obesity, it is possible that multiparametric MRI will become more useful considering transient elastography's unreliability and failed measurements associated with increased BMI. ${ }^{25}$ 
Moreover, it has been observed that the rate of uninterpretable results with transient elastography (due to fewer than 10 valid measurements) is $9.6 \%$, a value that could be an underestimation due to potential underreporting. ${ }^{28}$ The relationship between BMI and the prevalence of each stage of fibrosis has not been quantified, limiting any assessment of how the increased failure rate associated with obesity affects the diagnostic accuracy.

Controversy remains regarding the optimal cut-off values to diagnose advanced fibrosis using transient elastography as the cut-off values differ across aetiologies. This leads to variation in the interpretation of transient elastography results. ${ }^{26}$ Initial findings suggest that multiparametric MRI can quantify the severity of liver disease. ${ }^{27}$ This has implications in the monitoring and evaluation of liver disease progression. Currently, repeated liver biopsies are necessary to assess the stage liver disease. From a patient and payers' perspective, it would be preferable that the progression of liver disease be evaluated by a non-invasive method capable of assessing the stage of the disease rather than by an invasive and more costly liver biopsy. Since increasing disease activity may also occur in patients with simple steatosis, all patients with NAFLD should undergo periodic disease progression assessment with lifestyle modification advice if appropriate. ${ }^{9}$ The value of transient elastography in detecting early stages of liver disease is limited. Results of patients with low-stage grades of fibrosis $(\mathrm{F}<2)$ have been associated with significantly reduced reproducibility when compared with those of patients with marked fibrosis. ${ }^{9} 30$

The current decision analytic model aims to compare only the diagnostic pathways for patients presenting with suspected NAFLD. It does not consider the consequences of any diagnosis, either correct or incorrect, or failures to diagnose, with subsequent short-term and long-term disease progression and associated treatment outcomes. Patients with suspected NAFLD whose tests are within the normal range with either transient elastography or multiparametric MRI should subsequently be retested within a period of 1-2 years in order to capture any possible disease progression. The model does not follow the progression of liver disease in individuals. Rather, it presumes that the prevalence of the various levels of fibrosis in the population presenting with suspected fatty liver disease, and associated diagnostic outcomes, remain broadly the same whether patients be new or returning. A more comprehensive model could be developed to consider the longerterm progression of liver disease in individuals combined with the treatment outcomes associated with the diagnoses. This would need considerably more evidence, and the HTA was unable to identify robust cost and quality-adjusted life year estimates or data on treatment effectiveness to inform such a model ${ }^{28}$ Future research should attempt to address the shortcomings of currently available evidence for this patient population. Other non-invasive techniques are emerging such as MR elastography, which uses a vibration source to generate low-frequency mechanical waves in tissue. ${ }^{31-33}$ The wave information is processed allowing the quantitative assessment of the mechanical properties of tissue. The purpose of the current study was to evaluate the inclusion of multiparametric MRI in current diagnostic pathways for patients presenting with suspected NAFLD. MR elastography may be a valuable addition to currently used techniques and should be evaluated in further studies.

This model presumes that patients would not deviate from the best practice guidelines for diagnostic pathways. ${ }^{14}$ However, in practice, diagnosis and treatment initiation is often solely based on clinical judgement without biopsy. A recent survey observed that fewer than $25 \%$ of participating specialists performed liver biopsies to diagnose NASH, which diverges from guidelines and may leave NASH underdiagnosed in gastroenterology and hepatology clinics. ${ }^{34}$

Based on current practice, the reference standard for this model was liver biopsy, which is still regarded as the reference for differentiating steatosis from NASH, for staging hepatic fibrosis and for identifying NAFLD in patients with other chronic liver disease. ${ }^{35}$ However, this is an imperfect reference standard and has recently been considered that liver biopsy is not a suitable test for monitoring responses to therapy or for following disease progression. ${ }^{36}$ A UK national audit found that samples were insufficient for diagnosis in $71(2.04 \%)$ of 3472 cases. $^{37}$ Inadequate liver biopsies in which a focal lesion was present at imaging occurred in $82(7.1 \%)$ of 1162 biopsies and in $37(1.7 \%)$ of 2155 liver biopsies where a focal lesion was not present. ${ }^{37}$ The risk of excessive bleeding is about 1 in 500 to 1 in 1000 and the risk of death is about 1 in 10000 to 1 in $12000 .^{38}$ Although this risk is substantially lower than previous reports, it should be noted that transient elastography and multiparametric MRI have not been associated with any serious side effects. These aspects should be taken into account when modelling the long-term diagnostic pathways.

\section{CONCLUSION}

This study demonstrates that the inclusion of multiparametric MRI in the diagnostic pathway of NAFLD may lead to savings to the NHS if the model presumptions hold. Multiparametric MRI could be included either as an adjunct to or replacement of transient elastography, with both scenarios presenting savings compared with the current pathway to initial fatty liver diagnosis. In our model, the use of multiparametric MRI as an adjunct to transient elastography is predicted to more than halve the number of biopsies required. It is important to generate additional high-quality clinical evidence and cost data to develop the model further, and test its predictions. Current results suggest investment in evidence generation would have value. If these studies show that multiparametric MRI is able to match the diagnostic 
accuracy of liver biopsy to quantify disease progression, then there is the potential for it to replace biopsy for the diagnosis of liver fibrosis, with significant cost savings to the healthcare provider.

Acknowledgements The authors are grateful to Professor David Adams for his comments on an earlier version of this manuscript; George Bramley for managing the project, Zulian Liu for conducting literature searches and Melita Shirley for administrative support (all from University of Birmingham). The authors also thank Drs Rajarshi Banerjee, Michael Pavlides, Catherine Kelly and Professor Stefan Neubauer from Perspectum Diagnostics for information provided regarding multiparametric MRI.

Contributors All authors had an integral role in producing this manuscript and have made substantial contributions to the analysis (LB, RVD) and interpretation of data (LB, RVD), drafting (LB, RVD) and revision of the article (CC) and approving the final version of the manuscript (LB, RVD, CC).

Funding This manuscript presents independent research funded by a University of Birmingham Innovation Voucher awarded to Perspectum Diagnostics.

Competing interests None declared.

Provenance and peer review Not commissioned; externally peer reviewed.

Data sharing statement No additional data are available.

Open Access This is an Open Access article distributed in accordance with the Creative Commons Attribution Non Commercial (CC BY-NC 4.0) license, which permits others to distribute, remix, adapt, build upon this work noncommercially, and license their derivative works on different terms, provided the original work is properly cited and the use is non-commercial. See: http:// creativecommons.org/licenses/by-nc/4.0/

\section{REFERENCES}

1. Liang TJ. Hepatitis B: the virus and disease. Hepatology 2009;49: S13-21.

2. Tannapfel A, Denk H, Dienes HP, et al. Histopathological diagnosis of non-alcoholic and alcoholic fatty liver disease. Virchows Arch 2011;458:511-23.

3. Williams $\mathrm{R}$, Aspinall $\mathrm{R}$, Bellis $\mathrm{M}$, et al. Addressing liver disease in the UK: a blueprint for attaining excellence in health care and reducing premature mortality from lifestyle issues of excess consumption of alcohol, obesity, and viral hepatitis. Lancet 2014;384:1953-97.

4. Office for National Statistics. Mortality statistics: deaths registered in England and Wales (series DR), 2010. Newport, UK: Office for National Statistics, 2011

5. Younossi ZM, Stepanova M, Afendy M, et al. Changes in the prevalence of the most common causes of chronic liver diseases in the United States from 1988 to 2008. Clin Gastroenterol Hepatol 2011:9:524-30.

6. Finkelstein EA, Khavjou OA, Thompson $\mathrm{H}$, et al. Obesity and severe obesity forecasts through 2030. Am J Prev Med 2012;42:563-70.

7. Pais R, Pascale A, Fedchuck L, et al. Progression from isolated steatosis to steatohepatitis and fibrosis in nonalcoholic fatty liver disease. Clin Res Hepatol Gastroenterol 2011;35:23-8.

8. Pais R, Charlotte F, Fedchuk L, et al. A systematic review of follow-up biopsies reveals disease progression in patients with non-alcoholic fatty liver. J Hepatol 2013;59:550-6.

9. Wong VW, Wong GL, Choi PC, et al. Disease progression of non-alcoholic fatty liver disease: a prospective study with paired liver biopsies at 3 years. Gut 2010;59:969-74.

10. Ekstedt M, Franzén LE, Mathiesen UL, et al. Long-term follow-up of patients with NAFLD and elevated liver enzymes. Hepatology 2006;44:865-73.

11. Adams LA, Lymp JF, St Sauver J, et al. The natural history of nonalcoholic fatty liver disease: a population-based cohort study. Gastroenterology 2005;129:113-21.

12. Harrison SA, Torgerson S, Hayashi PH. The natural history of nonalcoholic fatty liver disease: a clinical histopathological study. Am J Gastroenterol 2003;98:2042-7.
13. Blachier M, Leleu H, Peck-Radosavljevic M, et al. The burden of liver disease in Europe: a review of available epidemiological data. $J$ Hepatol 2013;58:593-608.

14. European Association for the Study of the Liver (EASL); European Association for the Study of Diabetes (EASD); European Association for the Study of Obesity (EASO). EASL-EASD-EASO Clinical Practice Guidelines for the management of non-alcoholic fatty liver disease. J Hepatol 2016;64:1388-402.

15. Castera L, Pinzani M. Biopsy and non-invasive methods for the diagnosis of liver fibrosis: does it take two to tango? Gut 2010;59:861-6.

16. Afdhal NH. Fibroscan (transient elastography) for the measurement of liver fibrosis. Gastroenterol Hepatol 2012;8:605-7.

17. O'Shea RS, Dasarathy S, McCullough AJ, et al. Alcoholic liver disease. Hepatology 2010;51:307-28.

18. Poynard T, Lenaour G, Vaillant JC, et al. Liver biopsy analysis has a low level of performance for diagnosis of intermediate stages of fibrosis. Clin Gastroenterol Hepatol 2012;10:657-63.

19. Grant A, Neuberger J. Guidelines on the use of liver biopsy in clinical practice. British Society of Gastroenterology. Gut 1999;45 (Suppl 4): IV1-IV11.

20. Kan VY, Marquez Azalgara V, Ford JA, et al. Patient preference and willingness to pay for transient elastography versus liver biopsy: a perspective from British Columbia. Can J Gastroenterol Hepatol 2015;29:72-6.

21. Sandrin L, Fourquet B, Hasquenoph JM, et al. Transient elastography: a new noninvasive method for assessment of hepatic fibrosis. Ultrasound Med Biol 2003;29:1705-13.

22. Pavlov CS, Casazza G, Nikolova D, et al. Transient elastography for diagnosis of stages of hepatic fibrosis and cirrhosis in people with alcoholic liver disease. Cochrane Database Syst Rev 2015;1: CD010542.

23. Nascimbeni F, Lebray $P$, Fedchuk $L$, et al. Significant variations in elastometry measurements made within short-term in patients with chronic liver diseases. Clin Gastroenterol Hepatol 2015;13:763-71.

24. Foucher J, Castéra L, Bernard PH, et al. Prevalence and factors associated with failure of liver stiffness measurement using FibroScan in a prospective study of 2114 examinations. Eur J Gastroenterol Hepatol 2006;18:411-12.

25. Castéra L, Foucher J, Bernard PH, et al. Pitfalls of liver stiffness measurement: a 5 -year prospective study of 13,369 examinations. Hepatology 2010;51:828-35.

26. Wong GLH. Update of liver fibrosis and steatosis with transient elastography (Fibroscan). Gastroenterol Rep 2013;1:19-26.

27. Banerjee R, Pavlides M, Tunnicliffe EM, et al. Multiparametric magnetic resonance for the non-invasive diagnosis of liver disease. $J$ Hepatol 2014;60:69-77.

28. Crossan C, Tsochatzis EA, Longworth L, et al. Cost-effectiveness of non-invasive methods for assessment and monitoring of liver fibrosis and cirrhosis in patients with chronic liver disease: systematic review and economic evaluation. Health Technol Assess 2015;19:1-409, v-vi.

29. Curtis L. Unit costs of health and social care. Personal Social Services Research Unit, 2014. http://www.pssru.ac.uk

30. Fraquelli M, Rigamonti C, Casazza G, et al. Reproducibility of transient elastography in the evaluation of liver fibrosis in patients with chronic liver disease. Gut 2007;56:968-73.

31. Mariappan YK, Glaser KJ, Ehman RL. Magnetic resonance elastography: a review. Clin Anat 2010;23:497-511.

32. Venkatesh SK, Yin M, Ehman RL. Magnetic resonance elastography of liver: technique, analysis and clinical applications. J Magn Reson Imaging 2013;37:544-55.

33. Low G, Kruse SA, Lomas DJ. General review of magnetic resonance elastography. World J Radiol 2016;8:59-72.

34. Rinella ME, Lominadze Z, Loomba R, et al. Practice patterns in NAFLD and NASH: real life differs from published guidelines. Therap Adv Gastroenterol 2016;9:4-12.

35. Bedossa P, Patel K. Biopsy and noninvasive methods to assess progression of nonalcoholic fatty liver disease. Gastroenterology 2016;150:1811-22.e4.

36. Byrne CD, Targher G. EASL-EASD-EASO Clinical Practice Guidelines for the management of non-alcoholic fatty liver disease: is universal screening appropriate? Diabetologia 2016;59:1141-4.

37. Howlett DC, Drinkwater KJ, Lawrence D, et al. Findings of the UK national audit evaluating image-guided or image-assisted liver biopsy. Part I. Procedural aspects, diagnostic adequacy, and accuracy. Radiology 2012;265:819-31.

38. British-Liver-Trust.org.uk. Liver biopsy. http://www.british-liver-trust. org.uk/liver/liver-biopsy.html (assessed 26 Jan 2016). 